\title{
Tackling sleeplessness: Psychological treatment options for insomnia
}

\author{
This article was published in the following Dove Press journal: \\ Nature and Science of Sleep \\ 27 March 2010 \\ Number of times this article has been viewed
}

\author{
Natalie D Dautovich' \\ Joseph McNamara ${ }^{2}$ \\ Jacob MWilliams ${ }^{3}$ \\ Natalie J Cross ${ }^{4}$ \\ Christina S McCrae 3 \\ 'Department of Psychology, \\ ${ }^{2}$ Department of Psychiatry, \\ ${ }^{3}$ Department of Clinical and Health \\ Psychology, University of Florida, \\ Gainesville, FL, USA; ${ }^{4}$ Department of \\ Veterans Affairs Community Based \\ Outpatient Clinic, Greenville, NC, USA
}

\begin{abstract}
The purpose of the present paper is to review and summarize the research supporting nonpharmacologic treatment options for insomnia. The different treatment approaches are described followed by a review of both original research articles and meta-analyses. Meta-analytic reviews suggest that common nonpharmacologic approaches exert, on average, medium to large effect sizes on SOL, WASO, NWAK, SQR, and SE while smaller effects are seen for TST. Stimulus control therapy, relaxation training, and CBT-I are considered standard treatments for insomnia by the American Academy of Sleep Medicine (AASM). Sleep restriction, multicomponent therapy without cognitive therapy, paradoxical intention, and biofeedback approaches have received some levels of support by the AASM. Sleep hygiene, imagery training, and cognitive therapy did not receive recommendation levels as single (standalone) therapies by the AASM due to lack of empirical evidence. Less common approaches have been introduced (Internet-based interventions, bright light treatment, biofeedback, mindfulness, acupuncture, and intensive sleep retraining) but require further research. Brief and group treatments have been shown to be as efficacious as longer and individually-administered treatments. Considerations are presented for special populations, including older adults, children and teens, individuals from diverse cultural backgrounds, insomnia comorbid with other disorders, and individuals who are taking hypnotics.
\end{abstract}

Keywords: insomnia, nonpharmacologic, psychological, behavioral, treatments, sleep

\section{Introduction}

Insomnia, defined as difficulty initiating or maintaining sleep with impaired daytime functioning, is a prevalent complaint among the general population. Studies indicate that approximately $30 \%$ of the population report sleep disruption, while $10 \%$ report sleep disruption accompanied by daytime fatigue. ${ }^{1}$ Insomnia can be classified in several ways. For example it can be primary (not due to another sleep disorder or underlying psychiatric, medical, or substance abuse condition) or comorbid (occurring with another condition). It can also be classified as acute (ie, less than four weeks) or chronic (eg, greater than four weeks).

In addition to being a prevalent condition, the consequences of insomnia are significant and include increased risk of health problems, health-care utilization, work absenteeism, reduced productivity, and nonmotor-vehicle accidents. ${ }^{2}$ Based on a six-month period in 2003, Ozminkowski and colleagues estimated that the economic costs for those diagnosed with insomnia are greater than $\$ 1000$ more than those not diagnosed with insomnia. ${ }^{3}$

According to the National Institutes of Health, cognitive behavioral therapy (CBT-I) and pharmacotherapy (benzodiazepine receptor agonists) ${ }^{4}$ are the two
Correspondence: Christina S McCrae Department of Clinical and Health Psychology, University of Florida, PO Box 100165, Gainesville, FL 32610-0165, USA

$\mathrm{Tel}+\mathrm{I} 3522736053$

Email csmccrae@phhp.ufl.edu 
types of treatments that currently meet the criteria for use in the clinical management of insomnia. The purpose of the present paper is to review and summarize the research supporting nonpharmacologic treatment options for insomnia. The different treatment options will first be defined, followed by a review of common approaches and emerging/less common approaches, and treatment considerations for special populations. This paper will conclude with an evaluation of the overall effectiveness of nonpharmacologic interventions.

\section{Assessment of insomnia}

Researchers and clinicians have used a variety of objective and subjective techniques to assess sleep. Objective and subjective measures play different roles in both the research and clinical assessment of insomnia. Objective measurement through the use of polysomnography is useful within both clinical and research settings for ruling out occult sleep disorders such as sleep apnea. In particular, polysomnography can be useful for identifying sleep disordered breathing in older adults because self-reports of snoring and breathing cessation may not be as reliable in this population. ${ }^{5}$ Conversely, subjective measures, such as sleep diaries, are more important for the assessment of insomnia symptoms. Sleep diaries are a daily self-report measurement of sleep that requires individuals to document specific information regarding their previous night's sleep (eg, bedtime, number of minutes spent awake). Given the subjective nature of the insomnia complaint, a self-report measure is necessary to assess appropriately an individual's subjective sleep experience.

Research has been conducted to develop sufficient quantitative criteria to use self-report measures to diagnose insomnia in an effective manner. According to Lichstein and colleagues, the DSM-IV, ICSD, and ICD-10 do not offer sufficient quantitative criteria for diagnosing insomnia. ${ }^{6}$ They claim that this lack of a standardized set of quantitative criteria for evaluating self-reported sleep onset latency (SOL) and wake after sleep onset (WASO) is particularly problematic for research purposes because it produces undesirable variability in insomnia research. To address this issue, Lichstein and colleagues reviewed 61 clinical insomnia trials conducted during the 1980s and 1990s and used sensitivity-specificity analyses to identify the most empirically defensible quantitative criteria for insomnia. ${ }^{6}$ Based on their analyses, they recommend the following minimum insomnia diagnostic criteria for SOL or WASO of: (a) at least 31 minutes; (b) occurring at least three nights per week; (c) having occurred for at least six months.
While poor sleep is essential to diagnosing insomnia, daytime impairment is also needed for a diagnosis of insomnia. ${ }^{7}$ Lichstein and colleagues examined insomniacs' responses on five questionnaires that assess various aspects of daytime functioning. ${ }^{6}$ Based on their examination, they established cut-offs for each questionnaire. Specifically, according to Lichstein and colleagues, a person who surpasses the cutoff score on at least one of the following five questionnaires meets the daytime impairment criteria necessary for a diagnosis of insomnia.

It is important to note that the cutoffs recommended below are specific to assessing level of daytime impairment relative to insomnia. Each instrument has other established cutoffs for diagnosing the symptoms/disorder they were originally designed to assess. For example, the cutoff score on the Epworth Sleepiness Scale (ESS); (see below for more details) that indicates an individual has a level of daytime sleepiness consistent with a diagnosis of apnea ( $\geq 11)$ is different from the recommended cutoff ( $\geq 7.4$ ) for assessing insomnia-related daytime impairment. This distinction is particularly important, because true daytime sleepiness (the tendency to fall asleep in sedentary situations) is a hallmark symptom of apnea and can be useful in differentiating between insomnia and apnea. Specifically, apneics report higher tendencies for daytime sleepiness and are more likely to fall asleep; whereas insomniacs will frequently report daytime sleepiness, but further assessment typically indicates that their likelihood of actually falling asleep is relatively low (consistent with hyperarousal-related theories of insomnia). Polysomnographic assessment may be indicated for individuals who report a high level of likelihood of falling asleep during the day (eg, a score of $\geq 11$ ). While questionnaires can be useful in assessing daytime impairment, they are not always necessary because a thorough clinical sleep history interview can also be used to assess daytime impairment.

\section{Epworth Sleepiness Scale}

The ESS measures trait daytime sleepiness in everyday situations. ${ }^{8}$ Participants indicate how likely they are to fall asleep in eight common, quiet daytime activities over the previous two weeks. The possible range of scores is from 0 to 24 , with increasing scores indicating increasing daytime sleepiness. The recommended cutoff for the ESS is $\geq 7.4 .^{9}$

\section{Insomnia Impact Scale}

The Insomnia Impact Scale (IIS) is the most wide-ranging index of daytime functioning in insomnia available. ${ }^{10}$ The 
questionnaire contains 40 negative statements about the daytime impact of sleep. Respondents rate each item on a five-point scale to register their degree of agreement. The range of possible scores is from 40 to 200, with increasing scores indicating increasing impact of insomnia on daytime functioning. The recommended cutoff for the IIS is $\geq 125$. ${ }^{9}$

\section{Fatigue Severity Scale}

The Fatigue Severity Scale (FSS) comprises nine items that assess the intrusion of fatigue. ${ }^{11}$ Responses are averaged across the nine items, yielding a possible score range of 1-7. The cutoff for the FSS is $\geq 5.5$. $^{9}$

\section{Beck Depression Inventory}

The Beck Depression Inventory (BDI) is a widely used 21-item survey that measures negative affect, cognitions, and behavior. ${ }^{12}$ The recommended cutoff for the BDI is $\geq 10$. $^{9}$

\section{State-trait Anxiety Inventory Trait Scale (STAI)}

The State-trait Anxiety Inventory Trait Scale (STAI) is one of the most commonly used anxiety inventories. The STAI consists of 20 self-descriptive statements. ${ }^{13}$ A cutoff score of $\geq 37$ is recommended. ${ }^{9}$

\section{Methods}

A review of the literature was conducted to identify studies focused on nonpharmacologic treatments for insomnia. Articles published from 1972 to 2009 were included in the review. Search terms included: insomnia and treatment; stimulus control; paradoxical techniques; paradoxical intention; sleep restriction; sleep compression; sleep hygiene; and sleep education. The following search terms combined with insomnia were also used: nonpharmacologic therapy, behavior therapy, cognitive therapy, cognitive-behavioral therapy, psychotherapy, alternative medicine, relaxation, group therapy, telephone therapy, internet therapy, bright light therapy, and biofeedback. Where possible, meta-analyses are included in the review because they are considered the "gold-standard" of clinical evidence. Literature searches were conducted using the ISI Web of Science, PsychInfo, and PubMed databases, and Google Scholar.

Throughout this review, a number of sleep outcomes are described. The majority of outcomes are derived from sleep diary data because self-report data is the recommended standard for assessment of insomnia complaints. The sleep variables assessed by the studies include time taken to fall asleep (sleep onset latency; SOL), the number of times an individual awakens (number of awakenings, NWAK), time spent awake during the night after initial sleep onset (WASO), total time spent asleep (total sleep time; TST), efficiency of sleep defined as a ratio of time spent sleeping to time spent in the bed (sleep efficiency; SE), and the quality of sleep (sleep quality rating; SQR).

\section{Treatment components of psychological and behavioral interventions}

The purpose of this section is to outline and discuss the mechanics of common cognitive and behavioral treatments for insomnia. These interventions include stimulus control, sleep restriction, sleep compression, relaxation training, and cognitive therapy (summarized in Table 1).

Table I Summary of psychological and behavioral treatments for insomnia

\begin{tabular}{|c|c|}
\hline Treatment & Description \\
\hline Stimulus control & $\begin{array}{l}\text { Aims to restore the learned association } \\
\text { between the bedroom and sleeping. } \\
\text { Instructions often include using the } \\
\text { bedroom only for sleep (or sex), } \\
\text { getting out of bed if awake for } \\
\text { I5-20 minutes (and returning when } \\
\text { sleepy), avoiding napping, and setting } \\
\text { regular bed and wake times. }\end{array}$ \\
\hline Sleep restriction & $\begin{array}{l}\text { Consists of limiting the amount of } \\
\text { time spent in bed to the actual amount } \\
\text { of time spent sleeping by setting a } \\
\text { prescribed bed and wake time. Periodic } \\
\text { adjustments are made to the bed/wake } \\
\text { times depending on the level of sleep } \\
\text { efficiency. }\end{array}$ \\
\hline
\end{tabular}

Sleep compression

A variation on sleep restriction that involves a gradual reduction in the time spent in bed.

Relaxation

Used to reduce physical and mental tension. Common types include progressive muscle relaxation, autogenic training, imagery, and meditation.

Cognitive therapy Involves uncovering faulty underlying beliefs regarding sleep, providing alternative interpretations, and allowing the patient to consider their insomnia in a different way.

Multicomponent therapy

Note: Sleep education and sleep hygiene are often included as part of the multicomponent approach but have little empirical support as a stand-alone treatment. 


\section{Stimulus control}

Bootzin originated the concept of stimulus control for sleep in $1972,{ }^{14}$ and it has since enjoyed considerable success and confirmation as an effective monotherapy for insomnia. Stimulus control is based on the notion that with any behavior, one stimulus may elicit many responses depending on the history of conditioning. Stimuli of the bed and bedroom elicit associations of relaxation and sleep in healthy sleepers. While stimulus control dictates that the bed and bedroom should be used solely for sleep and sex, people with insomnia often have a history of engaging in many sleep-disruptive behaviors in the bedroom, including reading, eating, worrying, watching television, and engaging in arousing conversation. They often perceive that spending excessive time in or near the bed increases their chances of getting more sleep, when this actually weakens the stimulus-response relationship between being in bed and falling sleep. Over time, the stimuli of the bed and bedroom can become cues for anxiety and frustration associated with trying to fall asleep. Additionally, internal cues such as mind-racing and physiological arousal can become interoceptive cues for further arousal and sleep disruption. ${ }^{15}$ Stimulus control instructions often include using the bedroom only for sleep (or sex), getting out of bed if awake for 15 to 20 minutes (and returning only when sleepy), avoiding napping, and setting regular bed and wake times. Importantly, stimulus control is contraindicated in patients with mania, epilepsy, and parasomnias, or those who are at risk for falls. ${ }^{16}$

\section{Sleep restriction}

Sleep restriction therapy consists of limiting the amount of time spent in bed to the actual amount of time spent sleeping, such that these two values nearly mirror each other after treatment. ${ }^{17}$ Sleep diary data is used to establish the average amount of time-in-bed using data from the previous one or two weeks. A sleep efficiency percentage is computed by dividing the average time spent sleeping by the average time-in-bed and multiplying this number by 100 . Sleep efficiency is then used to determine the prescribed time-in-bed. During followup sessions, the timein-bed prescription is often titrated up or down based on sleep diary data from the previous week. Sleep restriction allows a slight sleep debt to accrue whereby on subsequent nights, patients often fall asleep more easily and experience more consolidated sleep. Sleep restriction therapy is contraindicated for patients with a history of mania, obstructive sleep apnea, seizure disorder, parasomnias, or those at risk for falls. ${ }^{16}$

\section{Sleep compression}

A variation on sleep restriction therapy is called sleep compression therapy. While sleep restriction therapy abruptly reduces the time the patient is allowed to spend in bed, sleep compression therapy decreases the time gradually (eg, timein-bed is reduced gradually over a five-week period rather than during one week ${ }^{18}$ ).

\section{Relaxation}

Relaxation training is useful in reducing the physical and mental tension reported by many patients with insomnia. These methods are especially useful with insomnia patients who evidence a great deal of hypervigilance or physical complaints that can interfere with adaptive sleep patterns. The most common types of relaxation for treating insomnia include progressive muscle relaxation, autogenic training, imagery, and meditation. ${ }^{16,19} \mathrm{~A}$ passive form of progressive muscle relaxation may be used that eliminates the tensing phase of this particular method. With all relaxation methods, the clinician instructs the patient to adapt a calm, passive attitude and to use relaxation on a consistent basis in order to elicit the parasympathetic response before going to bed and/or during awakenings after sleep onset. The clinician encourages the patient to be an active consumer in discovering the relaxation method(s) that are optimally effective, based on their own self-knowledge. Lastly, the importance of practice in eliciting the relaxation response is emphasized. Relaxation training often plays an important role in multicomponent approaches to the treatment of insomnia and has been shown to be effective in meta-analytic reviews (see discussion below).

\section{Paradoxical intention}

Paradoxical intention involves instructing the patient to attempt to stay awake while remaining in bed at night. The rationale behind this approach is that by attempting to stay awake, the stress and frustration associated with trying to fall asleep will be reduced.

\section{Imagery training}

To engage in imagery training, the patient selects a relaxing image or memory and invokes the image using multiple senses in order to create a sense of relaxation.

\section{Cognitive therapy}

Morin $^{20}$ and Harvey ${ }^{21}$ have provided models of cognitive therapy for insomnia. ${ }^{21,22}$ Cognitive therapy involves uncovering 
faulty underlying beliefs regarding sleep, providing alternative interpretations, and allowing the patient to consider their insomnia in a different way. ${ }^{23}$ Morin and colleagues ${ }^{23,24}$ have described five primary aims for cognitive therapy (see Table 2). These aims address cognitive distortions specific to insomnia that can be addressed through discussion with the therapist and by completion of thought records outside of sessions.

The clinician can also encourage time for constructive worry ${ }^{25}$ as a means of coping with "unfinished business" from the day. Here, the patient writes down their concerns and possible solutions to their concerns. This can be a means of providing some sense of calm and closure to life stressors before initiating sleep. The use of distraction with imagery has also been successful in managing unwanted pre-sleep thoughts. $^{26}$

\section{Multicomponent therapy}

When used to treat insomnia, CBT-I is an umbrella term for treatment packages that include three or more insomnia techniques. Although the exact components included in CBT-I vary, techniques that are commonly included are sleep hygiene, stimulus control, sleep restriction, cognitive therapy, and relaxation training (see above for a discussion of the individual components). Sleep hygiene is not discussed separately because it is rarely studied as a "stand-alone" treatment. As there is not sufficient evidence to support the effectiveness of sleep hygiene as a sole approach, we will discuss it within the context of a multicomponent approach. CBT-I is traditionally conducted following a thorough sleep history interview. Treatment occurs face-to-face over four to eight weekly sessions that are 60 to 90 minutes in length. Treatment can be conducted in individual or group format. Frequently, the clinician begins by providing the patient with sleep hygiene and sleep education information on the basic mechanics of sleep and sleep-disrupting practices. In terms of sleep education, the clinician provides information on the prevalence of insomnia and intrapersonal variability in the amount of sleep needed. Common insomnia-perpetuating factors such as increasing sleep opportunity by spending excessive time in bed and napping are explained. The clinician then highlights the notion that, fortunately, sleeppromoting skills can be relearned with effort and practice. While debate exists concerning the instructions that sleep hygiene includes, bedroom factors and the use of caffeine, nicotine, alcohol, exercise, and heavy meals late in the day are commonly included. ${ }^{27}$ Sleep hygiene and sleep education information are not recommended as stand-alone treatments for insomnia, because research does not support their efficacy when used as monotherapies. However, CBT-I protocols including sleep hygiene and sleep education have been shown to be efficacious.

Regardless of the specific technique or techniques used to treat a person with insomnia, treatment typically begins by instructing the patient how to complete a sleep diary and by informing them that they will complete the sleep

Table 2 Primary aims of cognitive therapy ${ }^{23,24}$

\begin{tabular}{|c|c|}
\hline Aim & Description \\
\hline $\begin{array}{l}\text { Addressing a misattribution } \\
\text { of consequences }\end{array}$ & $\begin{array}{l}\text { While patients may experience real daytime consequences of insomnia, fearing and anticipating these daytime } \\
\text { consequences amplifies perceived harm. Irritability, poor concentration, and inefficiency are related to sleep } \\
\text { duration and sleep quality, although other factors besides sleep also affect these things. Patients are instructed } \\
\text { to think rationally about the objective consequences of sleep loss. Worries over insomnia can be more } \\
\text { problematic than the insomnia itself. }\end{array}$ \\
\hline $\begin{array}{l}\text { Correcting unrealistic sleep } \\
\text { expectations }\end{array}$ & $\begin{array}{l}\text { Everyone has intra-individual variability in their sleep patterns from one night to another. The clinician } \\
\text { encourages the patient to be tolerant of his or her own variability in total sleep time and sleep quality across } \\
\text { nights. Also, patients are educated about the futility of comparing their sleep need to the sleep needs of others } \\
\text { as everyone's needs for sleep are unique. }\end{array}$ \\
\hline $\begin{array}{l}\text { Decreasing performance } \\
\text { anxiety and learned } \\
\text { helplessness }\end{array}$ & $\begin{array}{l}\text { Many patients try to increase feelings of control over their sleep by "trying harder" to fall asleep, although this } \\
\text { leads to difficulty with de-arousal that is necessary for sleep initiation. }\end{array}$ \\
\hline $\begin{array}{l}\text { Correcting misconceptions } \\
\text { about the causes } \\
\text { of insomnia }\end{array}$ & $\begin{array}{l}\text { Patients often attribute insomnia to external causes that cannot be controlled. However, insomnia always } \\
\text { involves some behavioral and psychological factors over which the patient can exert some control. Patients } \\
\text { should be encouraged to focus on those modifiable, internal causes and correlates, such as improving their } \\
\text { conditioning for adaptive sleep and improving sleep hygiene. }\end{array}$ \\
\hline
\end{tabular}


diary each day throughout the treatment period. Morin describes the order of events in the typical treatment session as reviewing the sleep diary and progress from the previous week, identifying problems encountered in home practice, negotiating strategies for better treatment adherence, introducing the new treatment component, presenting supportive didactic material, and reviewing homework assignments. $^{22}$

\section{Review of studies using common psychological and behavioral interventions}

Studies evaluating the effectiveness of common psychological and behavioral interventions (sleep hygiene, relaxation, stimulus control, sleep restriction/compression, cognitive therapy, and cognitive behavioral therapy) will be reviewed first. Several meta-analytic studies have been conducted to evaluate the effectiveness of nonpharmacologic interventions for insomnia. Meta-analytic studies compile results from multiple studies which enables the calculation of the effect sizes of different treatments. Throughout this review, effects sizes are primarily reported using Cohen's $d$, a measure of effect size thought of as how many standard deviations separate the means of two groups. Effect sizes are classified as minor (0.20), medium (0.50), and large $(0.80) .{ }^{28}$ The treatment outcomes for the meta-analyses were derived primarily from sleep diary data. Five meta-analytic studies were conducted between 1994 and 2006. The three most recent of the meta-analyses specifically focus on the treatment of older adults. Additionally, a comprehensive review of the literature was published by Morin and colleagues. ${ }^{29}$ Recent studies are also presented.

\section{Overall effectiveness of nonpharmacologic interventions}

Meta-analyses of the effectiveness of nonpharmacologic interventions have been conducted between $1994^{30}$ and $2006^{29}$ (see Table 3 ). The studies have covered the time period of 1973 to 2004. A variety of psychological interventions were included in the analyses, such as stimulus control, sleep restriction, relaxation therapy, paradoxical intention, biofeedback, sleep hygiene education, combined behavioral approaches, imagery training, cognitive-behavioral treatment, and multicomponent approaches. Statistically significant changes in sleep were found for SOL (effect sizes ranging from $d=0.41^{31}-0.88^{30}$ ), WASO (effect sizes ranging from $d=0.61^{31}-0.69^{32}$ ), TST (effect sizes ranging
Table 3 Summary of meta-analytic studies evaluating psychological and behavioral treatments

\begin{tabular}{llll}
\hline Reference & $\begin{array}{l}\text { Time period } \\
\text { reviewed }\end{array}$ & $\begin{array}{l}\text { Number of } \\
\text { studies } \\
\text { reviewed }\end{array}$ & Total sample \\
\hline 30 & $1974-1993$ & 59 & 2201 \\
33 & $1973-1993$ & 66 & 1907 \\
31 & $1966-1998$ & 13 & 308 \\
34 & $1966-2002$ & 6 & 274 \\
32 & $1966-2004$ & 23 & - \\
29 & $1998-2004$ & 37 & 2246 \\
\hline
\end{tabular}

from $d=0.15^{31}-0.49^{33}$ ), NWAK (effect sizes ranging from $\left.d=0.25^{31}-0.63^{33}\right)$, SE $\left(d=0.74^{32}\right)$ and SQR (effect sizes ranging from $d=0.79^{32}-0.94^{33}$; see Figure 1). In addition to studies reporting effect sizes, Montgomery and Dennis saw modest treatment effects for WASO (decrease of 22 minutes) and TST (increase of 14.6 minutes) and minimal gains for SOL (decrease of three minutes). ${ }^{34}$ Differential effects of the treatment approaches were found in some of the reviews, with the most effective treatments overall being stimulus control $^{30,33}$ and combination treatments, ${ }^{32,33}$ with lower effectiveness reported for paradoxical intention. ${ }^{33}$ In terms of the duration of treatment effects, clinical gains were maintained on average for $\operatorname{six}^{30,31}$ to eight ${ }^{33}$ months.

A study by Morin and colleagues reviewed the evidence for psychological and behavioral interventions for insomnia from studies within the time period of 1998 to $2004 .^{29}$ Using the criteria developed by the American Psychological Association, ${ }^{35}$ the authors identified the following five treatments as meeting the criteria for well-established treatments for insomnia: stimulus control therapy, relaxation training, paradoxical intention, sleep restriction, and CBT-I. The authors noted that there was a trend/preference for investigators to combine two or more interventions when treating insomnia. In particular, they noted that the most common approaches involved an educational (sleep hygiene), behavioral (stimulus control, sleep restriction, relaxation), and cognitive therapy component. ${ }^{29}$

\section{Recent studies}

Research conducted since the most recent meta-analyses supports the above conclusions. Morin and colleagues found that CBT-I therapy with adults aged 30 to 72 with persistent insomnia showed significant improvements in sleep diary SOL (-19.9 minutes), WASO (-68.7 minutes), and SE (+14.4\%). ${ }^{36}$ Additionally, participants showed significant improvements in polysomnography measured SOL 


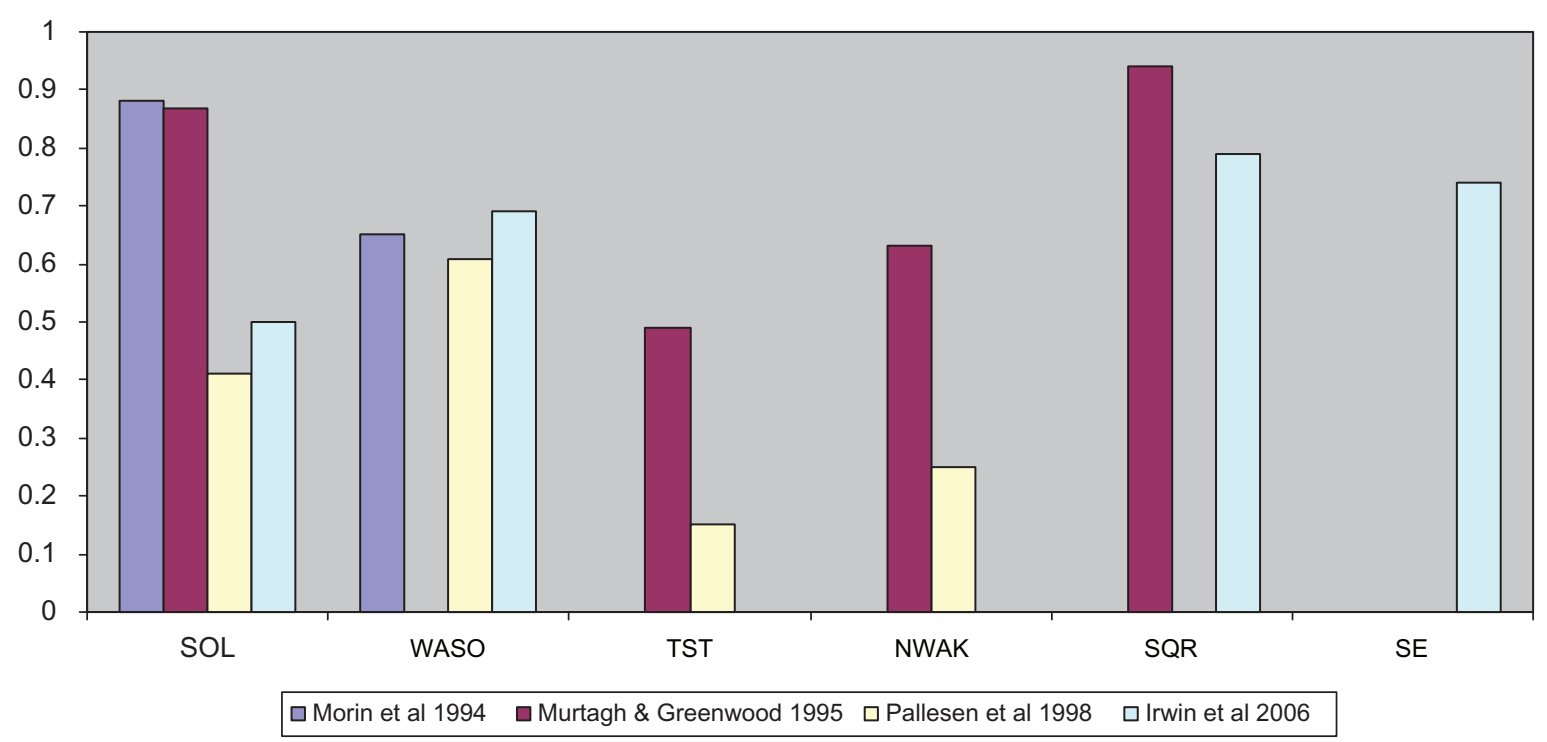

Figure I Average size of treatment effects (Cohen's $d$ ) on sleep variables from meta-analyses reporting effect sizes.

Abbreviations: SOL, sleep onset latency; WASO, wake time after sleep onset; TST, total sleep time; NWAK, number of awakenings; SQR, sleep quality rating; SE, sleep efficiency.

(-6.4 minutes), WASO ( -27.2 minutes), and SE (+5.5\%). Similarly, a recent study by Edinger and colleagues showed that CBT-I therapy significantly improved sleep outcomes (SOL [-19.8 minutes], WASO [-36 minutes], and SE [+12.1\%]) for those diagnosed with primary insomnia, with a mean age of 54.2 years. ${ }^{37}$

\section{Relative effectiveness of nonpharmacologic interventions}

While the above studies provide substantial evidence supporting the efficacy of nonpharmacologic interventions in general for insomnia, the relative efficacy of interventions remains unclear. While some studies have compared single therapy or combinations of treatments, there remains a lack of consensus as to the "best" single or combined therapies. In 2006, the American Academy of Sleep Medicine (AASM) released a report describing the evidential support for the psychological and behavioral treatment of insomnia. ${ }^{38}$ The AASM classified treatments according to the level of evidence with scores ranging from the highest level of evidence (level I for a randomized trial with low alpha and beta error) to the lowest (level $\mathrm{V}$ for a case series). Additionally, the AASM provided a level of recommendation for each treatment. Interventions could be classified as a "standard" recommendation (generally accepted strategy with level I or II evidence), "guideline" (moderate degree of clinical certainty with level II or III evidence), or "option" (uncertain clinical use with incon- clusive or conflicting evidence). Based on these criteria, stimulus control therapy, relaxation training, and CBT-I were recommended as standard treatments for insomnia. Sleep restriction, multicomponent therapy without cognitive therapy, paradoxical intention, and biofeedback received the guideline level of recommendation. Finally, sleep hygiene, imagery training, and cognitive therapy did not receive a recommendation level as a single therapy because there was insufficient evidence.

Interestingly, the AASM also stated that there was insufficient evidence to recommend one single therapy over another or to recommend a single therapy versus a combination of psychological and behavioral interventions. The relative efficacy of the single components of CBT-I has yet to be evaluated within a single study.

\section{Review of emerging/less common treatment approaches}

In addition to common psychological and behavioral treatments for insomnia, researchers and clinicians have also attempted to utilize new and nontraditional treatment approaches. While there is a dearth of empirical evidence supporting some of the newer treatments, they represent an innovative approach to the treatment of insomnia and consequently are reviewed here. These approaches include brief and group approaches as well as internet-based interventions, bright light treatments, techniques based on mindfulness meditation, and acupuncture. Additionally, biofeedback is 
reviewed. While it is not a new treatment, it represents a less commonly used approach.

\section{Brief and group approaches}

Traditionally, CBT-I protocols for insomnia last between six to 10 sessions and involve one-to-one meetings between the patient and clinician. These protocols require a large commitment on both the part of the clinician as well as patients. Because of these demands, researchers have sought to develop intervention protocols that require fewer sessions or personnel demands.

According to McCrae and colleagues, ${ }^{39}$ research has shown that both brief and group approaches for the treatment of insomnia can be as efficacious as traditional CBT-I approaches. Furthermore, brief and group treatments of insomnia have been utilized with a broad array of patient populations. $^{39}$

\section{Brief approaches}

According to Morin and colleagues, ${ }^{29}$ the average number of treatment sessions given for insomnia is 5.7 meetings. ${ }^{29}$ Based on their work, brief interventions are defined herein as treatment approaches that last for five or fewer sessions.

Numerous researchers have utilized four-session brief interventions. For example, McCrae and colleagues ${ }^{40}$ used a brief behavioral treatment with an older rural adult sample. The results showed improvements in SOL and SE. The treatment included stimulus control, sleep restriction, and passive relaxation. ${ }^{40}$ Lichstein and colleagues ${ }^{41}$ also used a four-session protocol to determine the efficacy of a brief approach for alleviating sleep complaints in a sample of older adults with secondary insomnia. Their treatment protocol consisted of sleep hygiene, stimulus control, and relaxation exercises. The results showed that the treatment led to significant improvements in WASO, SOL, SE, and SQR. ${ }^{41}$

Other researchers have examined the impact of even briefer interventions. Edinger and Sampson ${ }^{42}$ conducted a two-session cognitive-behavioral intervention. They found that participants showed a significant decrease in WASO, improved SE, and higher SQR. ${ }^{42}$ Another example is a study conducted by Chambers and Alexander that utilized a one-session approach. Their results indicated that SOL significantly decreased, WASO significantly decreased, and TST significantly increased. ${ }^{43}$ In contrast, other researchers have implemented group treatments for insomnia.

\section{Group approaches}

According to McCrae and colleagues, ${ }^{39}$ research has shown that group approaches have been successfully used with a wide array of populations. Researchers have conducted groups with as few as two people ${ }^{44}$ to as many as fifteen. ${ }^{45}$ The length of the treatment protocol ranges from three weekly sessions ${ }^{46}$ to eleven weekly sessions. ${ }^{47}$ Additionally these approaches have been utilized with adolescents ${ }^{44}$ and adults up to the age of 85 years. ${ }^{45}$

While group approaches vary in treatment approaches, most have contained at least one component of traditional cognitive-behavioral approaches. These approaches have been shown to improve sleep significantly. ${ }^{39}$ One of the additional benefits of a group approach is that the interactions among group members can strengthen the therapeutic benefit of group treatment. ${ }^{48}$

Briefer treatment protocols and the group administration of CBT-I provide useful alternatives to traditional treatments, because they reduce the time required of the clinician and are more appealing to patients. Additionally, studies of brief and group treatment of insomnia have demonstrated the utility of these approaches across a variety of ages and patient populations. Lastly, the effect sizes for both brief and group approaches are similar to the sizes of effect of traditional cognitive behavioral treatments of insomnia. ${ }^{39}$ These findings indicate that both brief and group treatment approaches are appropriate and as efficacious as traditional protocols. In addition to these brief and group treatment approaches, other treatment approaches have also been utilized.

\section{Other approaches}

Internet-based interventions, bright light treatments, biofeedback, and techniques based on mindfulness meditation and acupuncture have also been utilized to treat insomnia. For example, Ritterband and colleagues conducted a nine-week Internet intervention that included stimulus control, sleep hygiene, sleep restriction, relapse prevention, and cognitive restructuring. They found that the treatment led to significant improvements in WASO (decreased by 55\%) and SE (increased by $16 \%) .{ }^{49}$ Vincent and Lewycky conducted a six-week Internet intervention that found significant improvements in SQR $(+0.35)$ and significant declines in insomnia severity and daytime fatigue. ${ }^{50}$ Conversely, an Internet-based treatment intervention found that while the treatment group showed improvement on the Dysfunctional Beliefs and Attitudes about Sleep Scale, ${ }^{51}$ few significant differences between the treatment and control groups were seen in improvements in sleep. 
Lack and colleagues utilized a different technique to treat insomnia. They conducted a study that examined the impact of two bright light treatments on sleep. They found that the participants in the bright light treatment experienced a reduction in WASO and an increased TST. ${ }^{52}$

Ong and colleagues utilized another technique to treat insomnia. They integrated mindfulness meditation with CBT-I. The treatment lasted for six weeks and a significant correlation was found between the number of meditation sessions and arousal measures. The authors concluded that integrating mindfulness meditation led to significant improvement in insomnia symptoms, including total wake time ( -55 mins), SE (+9.08\%), and NWAK $(-0.99) .{ }^{53}$ Additionally, Ong and colleagues conducted a study with the same sample one year later and found that the treatment benefits were maintained..$^{54}$

Acupuncture is another technique that has been used for the treatment of insomnia. ${ }^{55}$ The authors found that both acupuncture and acupressure may help improve sleep quality when compared with control groups. Furthermore, Yeung and colleagues found that electro-acupuncture was helpful for increasing sleep efficiency. ${ }^{56}$ While these findings are encouraging, more evidence is needed to support the use of these techniques. ${ }^{55}$

Recently, researchers have begun to study another treatment approach known as intensive sleep retraining. This treatment approach is a condensed behavioral conditioning treatment occurring over a one-night period. The patient is allowed to sleep for brief periods of time (up to four minutes) before being awakened. The goal of the treatment is to decondition the insomnia arousal response to sleep onset, resulting in decreased sleep onset latency. Preliminary results suggest that intensive sleep retraining shows promise for decreasing SOL and increasing TST. ${ }^{57}$

While not a new approach, biofeedback is a treatment that has been used regularly in the past, but is less commonly used now. This technique provides feedback to patients to help them develop control over physiological responses that leads to a decrease in arousal. In the AASM report by Morgenthaler and colleagues it was concluded that this technique could be used with a moderate degree of clinical certainty. ${ }^{38}$ Additionally, Bootzin and Ryder's review of the literature on biofeedback revealed that biofeedback can be effective in the treatment of insomnia. ${ }^{58}$ They specifically examined electromyography (EMG), theta electroencephalography (EEG), and sensorimotor rhythm EEG. They found that EMG biofeedback can produce decreases in SOL, decreases in NWAK, decreases in WASO, and increased TST. However, they concluded that this technique was no more effective than relaxation training. According to Bootzin and Ryder's review, a few studies have shown that EEG biofeedback can be effective in the treatment of insomnia. However, few studies have utilized this technique in the last 30 years. Lastly, sensorimotor rhythm biofeedback has been shown to lead to an increase in the number and duration of sleep spindles, decreases in SOL, and an increase in the percentage of time spent in REM sleep. ${ }^{58}$

\section{Considerations for special populations}

The majority of empirical studies evaluating the effectiveness of psychological and behavioral interventions for insomnia have focused on healthy, community-dwelling adults with primary insomnia. Special consideration is required when implementing nonpharmacologic interventions with other populations, such as with older adults, children and teens, and individuals from diverse cultural backgrounds. Additionally, consideration must be taken when treating comorbid insomnia and when treating individuals who are taking hypnotics.

\section{Older adults}

Epidemiological research in the US suggests that the prevalence of insomnia increases across the lifespan among both men and women and is at its highest level late in life. ${ }^{59}$ Among older adults, those in their 70s are most likely to have maintenance insomnia (ie, difficulty maintaining sleep) ${ }^{59}$ In addition, older women are more likely than older men to have insomnia. ${ }^{59}$ Additionally, there is likely to be a high proportion of older individuals whose insomnia is comorbid in nature. ${ }^{60-63}$

Reviews and meta-analyses have supported the overall efficacy of psychological treatments for insomnia among older adults. ${ }^{31,64-68}$ Differences in the approaches to treating insomnia in older adults include differing expectations of sleep outcomes (eg, for sleep efficiency). For example, a goal of greater than $85 \%$ sleep efficiency is strived for rather than the $90 \%$ criteria used with younger adults. ${ }^{69}$ Also, difficulties may arise when trying to generalize results from research studies to the general older adult population because the majority of these studies were conducted in healthy, medication-free, community-dwelling and well-educated older adults. In reality, the presentation of insomnia among older adults is more likely to be comorbid with other disorders and these people are more likely to be using medications. ${ }^{69}$ In fact, the use of hypnotic medication by older adults is reported to be nearly five times the rate of younger adults. ${ }^{70,71}$ Consequently, clinicians using psychological treatments with older adults should be prepared for patients with a history of 
sleeping medication use and possible dependence or tolerance to sleeping medication. Given the multifaceted presentation of sleeping difficulties in older adults (eg, comorbid with other medical conditions and hypnotic use) multicomponent approaches should be considered to target most effectively the treatment needs of this population. For a complete discussion of the psychological treatment of insomnia in older adults see McCrae, Dzierzewski, and Kay, ${ }^{72}$ or Dzierzewski and colleagues' article concerning sleeplessness in older adults in the current volume.

\section{Children and teens}

Among children and teens, the definition and etiology of insomnia is much the same as among adults. However, the context surrounding the presenting complaint will be altered as the patient is often not the source of complaint. For children, sleeping difficulties are more likely to be voiced by the parent than the child. When assessing sleep difficulties in children, developmental stages must be taken into account to determine whether a sleep problem is normative. ${ }^{73}$ Additionally, the daytime impact of sleep disturbance among children may present differently than with adults, and is more likely to be characterized by behavior disturbance, irritability, and neuropsychological deficits. $^{74,75}$

Cognitive-behavioral treatment for sleep disturbance among children is based on many of the same principles as adults (ie, learned associations, physiological arousal). However, treatment of childhood insomnia requires the parent or guardian to implement much of the treatment, rather than the patient themselves. Parents play an essential role in developing and maintaining good sleep behaviors for their children, and especially among those with sleep disorders. ${ }^{76}$ While the techniques described in the current article apply more generally to adults, many of them may be useful for preteens/teens depending on the level of cognitive/emotional development of the individual and their degree of control over their own sleep/wake schedule. However, the techniques are not all equally generalizable to children. For example, stimulus control and sleep restriction, resulting in an initial decrease in sleep time, is likely to lead to mood and behavior problems rather than an increased sleep debt that will be helpful or therapeutic. Techniques specifically identified for treating childhood sleep disturbances include extinction and parent training (a preventative measure). ${ }^{77-79}$ An extinction treatment would advise the parent to ignore a child's bedtime tantrum for a specific period of time followed by a brief check-in by the parent. ${ }^{76}$ See the Mindell review ${ }^{76}$ for further evaluation of empirically supported treatments for childhood sleep disturbances.

\section{Cultural differences}

While there are known differences in the rates and presentation of sleep difficulties by different cultural groups, there has been a severe lack of research on cultural/ethnic differences in response to psychological treatments for insomnia. In terms of the presentation of sleep difficulties, older adult African-Americans are reported to have lower rates of insomnia than Caucasians. ${ }^{80-82}$ Caucasian and European American participants have reported fewer complaints of daytime napping than minority groups in the US, while Caucasian individuals tend to report higher rates of sleep medication use than African-American individuals. ${ }^{83-85}$ To date, there are no treatment studies that directly compare the treatment response of African-Americans and Caucasians. A comprehensive review of sleep literature pertaining to African-Americans has been done by Lichstein et al. ${ }^{9}$ Epidemiological studies from around the world have estimated the prevalence and incidence of chronic insomnia to be from $5 \%$ to $20 \%$ in local populations (Japan, South Korea, Malaysia, Belgium, Germany, Great Britain, Ireland, Sweden, and the US). ${ }^{86-91}$

Comparing samples from Japan and Western countries indicates that the prevalence, risk factors, and role of cognitive processes in the maintenance of insomnia are almost identical between these cultures. ${ }^{88,90,92-94}$ Differences have been reported in some of the perpetuating behaviors of insomnia, where Japanese participants were found to be more likely to spend time engaging in nocturnal activities. ${ }^{92,95}$

\section{Comorbid insomnia}

Most research has studied insomnia in isolation, known as primary insomnia. Insomnia symptoms related to another physical, psychological, or drug-induced condition were considered secondary to the primary diagnosis and referred to as "secondary insomnia". Recently, however, the National Institutes of Heath State of the Science Conference on Manifestation and Management of Chronic Insomnia in Adults (2005) recommended that insomnia classification should focus on comorbid insomnia diagnosis, rather than secondary insomnia. The classification of insomnia as comorbid rather than as secondary to other disorders is important, given how insomnia develops. According to 
the behavioral model of insomnia proposed by Spielman and colleagues, insomnia can develop in response to both predisposing and precipitating factors, and be maintained due to perpetuating factors. ${ }^{96}$ Predisposing characteristics such as hyperarousal can place an individual at increased risk for developing insomnia. Precipitating factors, such as disrupted sleep due to another illness, can instigate an acute insomnia episode. Finally, perpetuating factors include changes in behavior such as lying awake in bed while ill which can lead to maladaptive behaviors that prolong the insomnia. ${ }^{96}$ Consequently, while an individual may develop insomnia concomitant with or due to another disorder, insomnia can be perpetuated due to maladaptive behaviors and continue to require treatment in the absence of the comorbid illness. ${ }^{97}$

In recent years, interest has increased in evaluating the efficacy of psychological treatments for comorbid insomnia. A complete review of this literature is beyond the scope of this article but a brief synopsis is provided. Case studies have shown that cognitive and behavioral treatments improved insomnia among people with cancer, ${ }^{98}$ chronic pain, ${ }^{99,100}$ depression and pain, ${ }^{101}$ hemophilia, ${ }^{102}$ psychiatric disorders, ${ }^{103}$ and multiple medical problems. ${ }^{104}$ Randomized empirical studies have explored the effects of cognitive and behavioral therapies for insomnia and found them to be efficacious among those with chronic pain, ${ }^{105-107}$ fibromyalgia, ${ }^{107}$ cancer, ${ }^{108-110}$ early stage Alzheimer's disease, ${ }^{111}$ alcohol dependence, ${ }^{112}$ and older adults with medical diagnoses and psychiatric diagnoses ${ }^{37}$ A review of the diagnosis and treatment of comorbid/secondary insomnia has been done by McCrae and Lichtein. ${ }^{113}$ In addition to this research, McCrae and colleagues are currently conducting several NIH-funded studies examining the efficacy of CBT-I in patients experiencing chronic pain, patients with cardiac disease who have implantable cardioverter defibrillators, and patients with gynecologic cancers undergoing chemotherapy. The study examining cognitive-behavioural therapy in patients with chronic pain uses cognitive-behavioral interventions specific to insomnia (CBT-I) and chronic pain (CBT-P) to examine the causal links between sleep and pain. Additionally, the study is collecting neuroimaging data pre- and post-CBT-I to examine the neural mechanisms by which CBT-I interventions could interrupt the development and maintenance of increased sensitivity to pain. The study examining sleep in patients with implantable cardioverter defibrillators is collecting data on the prevalence of sleep disorders in these patients. Currently, information regard- ing the specific types of sleep disorders experienced by this population is not available. In addition to examining the prevalence of sleep disorders, this study is testing the effectiveness of a brief CBT-I intervention specifically designed to improve sleep in patients with an implantable cardioverter defibrillator. This research will also assess the cognitive functioning of these patients across the sleep disorders, including insomnia, apnea, etc. A third ongoing study is examining the effectiveness of CBT-I among women with gynecologic cancers undergoing chemotherapy. The study is using a multicomponent intervention with the goals of improving sleep and pain, and reducing cortisol levels in these women.

\section{Insomnia and use of hypnotics}

While older adults report the highest rates of hypnotic use, younger adults also report high levels of hypnotics used to treat sleep problems. ${ }^{72,114}$ Not surprisingly, individuals diagnosed with insomnia report higher rates of hypnotic use than those with undiagnosed sleep problems. As medication is often the first line of treatment, it is highly likely that individuals presenting for nonpharmacologic treatment of insomnia will have a history of pharmacologic treatment for insomnia. Data suggest that the combination of carefully administered CBT-I and pharmacologic treatment should result in a beneficial interaction. ${ }^{115}$ However, there are insufficient data at this time to make any conclusions. ${ }^{116}$ Research has shown that the use of CBT-I techniques (stimulus control, sleep hygiene, and relaxation) among hypnotic-dependent younger and older adults with insomnia can lead to statistically and clinically significant improvements in sleep and to reduced medication usage when compared with placebo. ${ }^{117,118}$

Whether to withdraw a patient from hypnotics over the course of psychological treatment or to maintain the patient's medications is often unclear because of concerns about the withdrawal process possibly resulting in worsened insomnia, often referred to as "rebound insomnia". A number of studies suggest that CBT-I is an efficacious technique to facilitate withdrawal from benzodiazepines and third-generation hypnotics among hypnotic-dependent younger and older adults. ${ }^{70,119-121}$ Patients are likely to experience a brief worsening of their insomnia symptoms but this is likely to subside within a few weeks. It is helpful to prepare patients for the side effects of withdrawal prior to beginning the withdrawal process, to monitor for symptoms of withdrawal throughout treatment, and to withdraw hypnotics gradually to ease the transition. 


\section{Conclusion}

Various psychological and behavioral interventions have been studied as treatments for insomnia. Meta-analyses suggest that common nonpharmacologic approaches exert, on average, a medium to large effect size on sleep variables such as SOL, WASO, NWAK, SQR, and SE (see Figure 1). Less significant effects are seen for TST which is understandable given that sleep treatments such as sleep restriction may curtail the total amount of time spent sleeping. ${ }^{122}$ Additionally, the AASM has concluded that stimulus control therapy, relaxation training, and CBT-I can be considered standard treatments for insomnia. Sleep restriction, multicomponent therapy without cognitive therapy, paradoxical intention, and biofeedback have received some levels of support. Finally, sleep hygiene, imagery training, and cognitive therapy did not receive a recommendation level as a single therapy as there was insufficient evidence.

In addition to the more common approaches to treating insomnia, research has evaluated emerging or less common approaches. Brief and group treatments have been shown to be as efficacious as longer and individuallyadministered treatments. Additionally, evidence is promising for emerging/less common treatments such as Internetbased interventions, bright light treatment, biofeedback, mindfulness, acupuncture, and intensive sleep retraining. Additional research, with randomized controlled trials, is required to support the efficacy of these approaches for treating insomnia. Finally, special consideration is required when implementing nonpharmacologic interventions with certain populations such as with older adults, children and teens, insomnia comorbid with another disorder, and insomnia with hypnotic use.

Future directions include examining the longevity of treatment effects, examining treatment effects with diverse samples, and recruiting "ecologically valid" samples consisting of individuals who experience comorbid disorders and other health complaints.

\section{Disclosures}

The authors report no conflict of interest in this work.

\section{References}

1. Ohayon MM. Epidemiology of insomnia: What we know and what we still need to learn. Sleep Med Rev. 2002;6(2):97-111.

2. Daley M, Morin CM, LeBlanc M, Gregoire JP, Savard J, Baillargeon L. Insomnia and its relationship to health-care utilization, work absenteeism, productivity and accidents. Sleep Med. 2009;10(4): $427-438$.
3. Ozminkowski RJ, Wang SH, Walsh JK. The direct and indirect costs of untreated insomnia in adults in the United States. Sleep. 2007;30(3):263-273.

4. National Institutes of Health. State of the Science Conference Statement - Manifestations and management of chronic insomnia in adults. Sleep. 2005;28(9):1049-1057.

5. Young T, Shahar E, Nieto FJ, et al. Predictors of sleep-disordered breathing in community-dwelling adults - The sleep heart health study. Arch Intern Med. 2002;162(8):893-900.

6. Lichstein KL, Durrence HH, Taylor DJ, Bush AJ, Riedel BW. Quantitative criteria for insomnia. Behav Res and Ther. 2003;41(4): 427-445.

7. American Psychiatric Association. Diagnostic and Statistical Manual of Mental Disorders. 4th ed. Washington, DC: American Psychiatric Association; 2000.

8. Johns MW. A new method for measuring daytime sleepiness: The Epworth sleepiness scale. Sleep. 1991;14:5.

9. Lichstein KL, Durrence HH, Riedel BW, Taylor DJ, Bush AJ. Epidemiology of Sleep: Age, gender, and ethnicity. Hillsdale, NJ: Lawrence Erlbaum Associates, Inc.; 2004.

10. Hoelscher TJ, Ware JC, Bond T. Initial validation of the Insomnia Impact Scale. Sleep Res. 1993;22:149.

11. Krupp LB, LaRocca NG, Muir-Nash J, Steinberg AD. The Fatigue Severity Scale: Application to patients with multiple sclerosis and systemic lupus erythematosus. Arch of Neurol. 1989;46(10): 1121-3.

12. Beck AT, Steer RA. Beck Depression Inventory. Orlando, FL: Psychological Corporation; 1987.

13. Spielberger CD, Gorsuch RL, Lushene R, Vagg PR, Jacobs GA. StaitTrait Anxiety Inventory, Form Y. Palo Alto, CA: Consulting Psychologists Press; 1983.

14. Bootzin RR. A stimulus control treatment for insomnia. Proceedings of the 80th Annual Convention of the American Psychological Association. 1972:395-396.

15. Bootzin RR, Epstein DR. Stimulus control. In: Lichstein KL, Morin CM, editors. Treatment of Late-life Insomnia. Thousand Oaks, CA: Sage Publications; 2000.

16. Perlis ML, Jungquist C, Smith MT, Posner D. Cognitive Behavioral Treatment of Insomnia. New York, NY: Springer; 2005.

17. Spielman AJ, Saskin P, Thorpy MJ. Treatment of chronic insomnia by restriction of time in bed. Sleep. 1987;10(1):45-56.

18. Lichstein KL, Nau SD, McCrae CS, Stone KC. Psychological and behavioral treatments for secondary insomnias. In: Kryger MH, Roth T, Dement WC, editors. Principles and Practice of Sleep Medicine. Philadelphia, PA: Saunders; 2005.

19. Lichstein KL. Relaxation. In: Lichstein KL, Morin CM, editors. Treatment of Late-life Insomnia. Thousand Oaks, CA: Sage Publications; 2000.

20. Morin CM. Insomnia. Psychological assessment and management. New York, NY: Guilford Press; 1993.

21. Harvey AG. A cognitive model of insomnia. Behav Res and Ther. 2002;40(8):869-893.

22. Morin CM. A cognitive-behavioral conceptualization of insomnia. In: Insomnia: Psychological assessment and management. New York, NY: Guilford Press; 1993.

23. Morin CM, Espie CA. Insomnia: A clinical guide to assessment and treatment. New York, NY: Kluwer Academic/Plenum; 2003.

24. Belanger L, Savard J, Morin CM. Clinical management of insomnia using cognitive therapy. Behav Sleep Med. 2006;4(3):179-202.

25. Carney CE, Waters WF. Effects of a structured problem-solving procedure on pre-sleep cognitive arousal in college students with insomnia. Behav Sleep Med. 2006;4(1):13-28.

26. Harvey AG, Payne S. The management of unwanted pre-sleep thoughts in insomnia: Distraction with imagery versus general distraction. Behav Res Ther. 2002;40(3):267-277.

27. Morin CM, Espie CA, editors. Insomnia: A clinical guide to assessment and treatment. New York, NY: Springer; 2004. 
28. Cohen J. Statistical power analysis for the behavioral sciences. New York: Academic Press; 1977.

29. Morin CM, Bootzin RR, Buysse DJ, Edinger JD, Espie CA, Lichstein KL. Psychological and behavioral treatment of insomnia: Update of the recent evidence (1998-2004). Sleep. 2006;29(11):1398-1414.

30. Morin CM, Culbert JP, Schwartz SM. Nonpharmacological interventions for insomnia - A meta-analysis of treatment efficacy. Am J Psychiatry. 1994;151(8):1172-1180.

31. Pallesen S, Nordhus IH, Kvale G. Nonpharmacological interventions for insomnia in older adults: A meta-analysis of treatment efficacy. Psychotherapy. 1998;35(4):472-482.

32. Irwin MR, Cole JC, Nicassio PM. Comparative meta-analysis of behavioral interventions for insomnia and their efficacy in middleaged adults and in older adults 55+ years of age. Health Psychol. 2006;25(1):3-14.

33. Murtagh DRR, Greenwood KM. Identifying effective psychological treatments for insomnia - a meta-analysis. $J$ of Consult and Clin Psychol.1995;63(1):79-89.

34. Montgomery P, Dennis J. A systematic review of non-pharmacological therapies for sleep problems in later life. Sleep Med Rev. 2004;8(1): 47-62.

35. Chambless DL, Hollon SD. Defining empirically supported therapies. J Consult and Clin Psychol. 1998;66:7-18.

36. Morin CM, Vallieres A, Guay B, et al. Cognitive behavioral therapy, singly and combined with medication, for persistent insomnia: A randomized controlled trial. JAMA. 2009;301(19):2005-2015.

37. Edinger JD, Olsen MK, Stechuchak KM, et al. cognitive behavioral therapy for patients with primary insomnia or insomnia associated predominantly with mixed psychiatric disorders: A randomized clinical trial. Sleep. 2009;32(4):499-510.

38. Morgenthaler T, Kramer M, Alessi C, et al. Practice parameters for the psychological and behavioral treatment of insomnia: An update. An American Academy of Sleep Medicine Report. Sleep. Nov 2006;29(11):1415-1419.

39. McCrae CS, Dautovich ND, Dzierzewski JM. Short-term and group approaches. In: Sateia MJ, Buysse DJ, editors. Insomnia: Diagnosis and treatment. New York: Informa Healthcare USA, Inc.; 2010.

40. McCrae CS, McGovern R, Lukefahr R, Stripling AM. Research evaluating brief Behavioral sleep treatments for rural elderly (RESTORE): A preliminary examination of effectiveness. Am J Geriatr Psychiatry. 2007;15(11):979-982.

41. Lichstein KL, Wilson NM, Johnson CT. Psychological treatment of secondary insomnia. Psychol Aging. 2000;15(2):232-240.

42. Edinger JD, Sampson WS. A primary care "friendly" cognitive behavioral insomnia therapy. Sleep. 2003;26(2):177-182.

43. Chambers MJ, Alexander SD. Assessment and prediction of outcome for a brief behavioral insomnia treatment program. J Behav Ther Exp Psychiatry. 1992; Dec 23(4):289-97.

44. Bootzin RR, Stevens SJ. Adolescents, substance abuse, and the treatment of insomnia and daytime sleepiness. Clin Psychol Rev. 2005;25(5): 629-644.

45. Constantino M, Manber R, Ong J, Juo T, Huang J, Arnow B. Patient expectations and therapeutic alliance as predictors of outcome in group cognitive-behavioral therapy for insomnia. Behav Sleep Med. 2007;5(3):210-228.

46. Borkovec TD, Steinmar.Sw, Nau SD. Relaxation training and single-item desensitization in group treatment of insomnia. J Behav Ther Exp Psychiatry. 1973;4(4):401-403.

47. Schramm E, Hohagen F, Backhaus J, Lis S, Berger M. Effectiveness of a multicomponent group treatment for insomnia. Behav Cogn Psychother. 1995;23(2):109-127.

48. Verbeek I, Konings G, Aldenkamp A, Declerck A, Klip E. Cognitive behavioral treatment in clinically referred chronic insomniacs: Group versus individual treatment. Behav Sleep Med. 2006;4(3):135-151.

49. Ritterband LM, Thorndike FP, Gonder-Frederick LA, et al. Efficacy of an internet-based behavioral intervention for adults with insomnia. Arch Gen Psychiatry. 2009;66(7):692-698.
50. Vincent N, Lewycky S. Logging on for better sleep: RCT of the effectiveness of online treatment for insomnia. Sleep. 2009;32(6): $807-815$.

51. Morin C, Stone J, Trinkle D, Mercer J, Remsberg S. Dysfunctional beliefs and attitudes about sleep among older adults with and without insomnia. Psychol Aging. 1993;8:463-467.

52. Lack L, Wright H, Kemp K, Gibbon S. The treatment of earlymorning awakening insomnia with 2 evenings of bright light. Sleep. 2005;28(5):616-623.

53. Ong JC, Shapiro SL, Manber R. Combining mindfulness meditation with cognitive-behavior therapy for insomnia: A treatment-development study. Behav Ther. 2008;39(2):171-182.

54. Ong JC, Shapiro SL, Manber R. Mindfulness meditation and cognitive behavioral therapy for insomnia: A naturalistic 12-month follow-up. EXPLORE: The Journal of Science and Healing. 2009;5(1): $30-36$.

55. Cheuk DKL, Yeung WF, Chung KF, Wong V. Acupuncture for insomnia. Cochrane Database Syst Rev. 2007;18(3):CD005472.

56. Yeung WF, Chung KF, Zhang SP, Yap TG, Law ACK. Electroacupuncture for primary insomnia: A randomized controlled trial. Sleep. 2009;32(8):1039-1047.

57. Harris J, Lack L, Wright H, Gradisar M, Brooks A. Intensive sleep retraining treatment for chronic primary insomnia: A preliminary investigation J Sleep Res. 2007;16:276-284.

58. Bootzin RR, Rider SP, editors. Behavioral techniques and biofeedback for insomnia. In: Pressman MR, Orr WC, editors. Understanding Sleep: The evaluation and treatment of sleep disorders. Washington, DC: American Psychological Association; 1997.

59. Lichstein KL, Durrence H, Riedel BW, Taylor DJ, Bush A. Epidemiology of sleep: Age, gender, and ethnicity. Mahwah, NJ: Lawrence Erlbaum Associates; 2004.

60. Foley D, Ancoli-Israel S, Britz P, Walsh J. Sleep disturbances and chronic disease in older adults: Results of the 2003 National Sleep Foundation Sleep in America survey. J Psychosom Res. 2004;56(5): 497-502.

61. Newman AB, Enright RL, Manolio TA, Haponik EE, Wahl PW. Sleep disturbance, psychosocial correlates, and cardiovascular disease in 5201 older adults: The cardiovascular health study. J Am Geriatr Soc. 1997;45(1):1472-1478.

62. Ohayon MM, Carskadon MA, Guilleminault C, Vitiello MV. Meta-analysis of quantitative sleep parameters from childhood to old age in healthy individuals: developing normative sleep values across the human lifespan. Sleep. 2004;27(7):1255-1273.

63. Vitiello MV, Moe KE, Prinz PN. Sleep complaints cosegregate with illness in older adults: Clinical research informed by and informing epidemiological studies of sleep. J Psychosom Res. 2002;53(1):555-559.

64. Irwin MR, Cole JC, Nicassio PM. Comparative meta-analysis of behavioral interventions for insomnia and their efficacy in middleaged adults and in older adults 55+ years of age. Health Psychol. 2006;25(1):3-14.

65. Morin CM, Mimeault V, Gagne A. Nonpharmacological treatment of late-life insomnia. J Psychosom Res. 1999;46(2):103-116.

66. Morin CM, Hauri PJ, Espie CA, Spielman AJ, Buysse DJ, Bootzin RR. Nonpharmacologic treatment of chronic insomnia. An American Academy of Sleep Medicine review. Sleep. 1999;22(8): 1134-1156.

67. Murtagh DR, Greenwood KM. Identifying effective psychological treatments for insomnia: A meta-analysis. J Consult Clin Psychol. 1995;63(1):79-89.

68. Nau SD, McCrae CS, Cook KG, Lichstein KL. Treatment of insomnia in older adults. Clin Psychol Rev. 2005;25(5):645-672.

69. Lichstein KL, Morin CM. Treatment of Late-Life Insomnia. Thousand Oaks, CA: Sage Publications; 2000.

70. Lichstein KL, Johnson RS. Relaxation for insomnia and hypnotic medication use in older women. Psychol Aging. 1993;8(1): $103-111$. 
71. Quera-Salva MA, Orluc A, Goldenberg F, Guilleminault C. Insomnia and use of hypnotics: Study of a French population. Sleep. 1991;14(5):386-391.

72. McCrae CS, Dzierzewski J, Kay D. Treatment of late-life insomnia. Sleep Med Clin. 2009;4:593-604.

73. Owens JA. Pediatric insomnia. Sleep Med Clin. 2006;1:12.

74. Randazzo AC, Muehlbach MJ, Schweitzer PK, Walsh JK. Cognitive function following acute sleep restriction in children ages 10-14. Sleep.1998;21(8):861-868.

75. Smedje H, Broman JE, Hetta J. Associations between disturbed sleep and behavioural difficulties in 635 children aged six to eight years: A study based on parents' perceptions. Eur Child Adolesc Psychiatry. 2001;10(1):1-9.

76. Mindell JA. Empirically supported treatments in pediatric psychology: Bedtime refusal and night wakings in young children. J Pediatr Psychol. 1999;24(6):465-481.

77. Wolfson A, Lacks P, Futterman A. Effects of parent training on infant sleeping patterns, parents' stress, and perceived parental competence. J Consult Clin Psychol. 1992;60(1):41-48.

78. Adair R, Zuckerman B, Bauchner H, Philipp B, Levenson S. Reducing night waking in infancy: A primary care intervention. Pediatrics. 1992;89(4 Pt 1):585-588.

79. Kerr SM, Jowett SA, Smith LN. Preventing sleep problems in infants: A randomized controlled trial. J Adv Nurs. 1996;24(5): 938-942.

80. Blazer DG, Hays JC, Foley DJ. Sleep complaints in older adults: A racial comparison. J Gerontol A Biol Sci Med Sci. 1995;50(5):M280-284.

81. Foley DJ, Monjan AA, Brown LS, Simonsick EM, Wallace RB, Blazer DG. Sleep complaints among elderly persons: An epidemiologic study of three communities. Sleep. 1995;18(6):425-432.

82. Jean-Louis G, Magai CM, Cohen CI, Zizi E, von Gizycki H, DiPalma J. Ethnic differences in self-reported sleep problems in older adults. Sleep. 2001;24(8):926-933.

83. Jean-Louis G, Magai C, Casimir GJ, et al. Insomnia symptoms in a multiethnic sample of American women. J Womens Health (Larchmt). 2008;17(1):15-25

84. Qureshi AI, Giles WH, Croft JB, Bliwise DL. Habitual sleep patterns and risk for stroke and coronary heart disease: A 10-year follow-up from NHANES I. Neurology. 1997;48(4):904-911.

85. Ancoli-Israel S, Klauber MR, Stepnowsky C, Estline E, Chinn A, Fell R. Sleep-disordered breathing in African-American elderly. Am J Respir Crit Care Med. 1995;152(6 Pt 1):1946-1949.

86. Chevalier H, Los F, Boichut D, et al. Evaluation of severe insomnia in the general population: Results of a European multinational survey. J Psychopharmacol. 1999;13(4 Suppl 1):S21-24.

87. Ohayon MM, Hong SC. Prevalence of insomnia and associated factors in South Korea. J Psychosom Res. 2002;53(1):593-600.

88. Tachibana H, Izumi T, Honda S, Takemoto TI. The prevalence and pattern of insomnia in Japanese industrial workers: Relationship between psychosocial stress and type of insomnia. Psychiatry Clin Neurosci. 1998;52(4):397-402.

89. Morphy H, Dunn KM, Lewis M, Boardman HF, Croft PR. Epidemiology of insomnia: A longitudinal study in a UK population. Sleep. 2007;30(3):274-280.

90. Kim K, Uchiyama M, Okawa M, Liu X, Ogihara R. An epidemiological study of insomnia among the Japanese general population. Sleep. 2000;23(1):41-7.

91. Zailinawati A, Ariff K, Nurjahan M, Teng C. Epidemiology of insomnia in Malaysian adults: a community-based survey in 4 urban areas. Asia Pac J Public Health. 2008;20(3):224-233.

92. Harvey A, Gregory A, Bird C. The role of cognitive processes in sleep disturbance: A comparison of Japanese and English university students. Behav Cogn Psychother. 2002;30:259-270.

93. Kageyama T, Kabuto M, Nitta H, et al. A population study on risk factors for insomnia among adult Japanese women: A possible effect of road traffic volume. Sleep. 1997;20(11):963-971.
94. Motohashi Y, Takano T. Sleep habits and psychosomatic health complaints of bank workers in a megacity in Japan. J Biosoc Sci. 1995;27(4):467-472.

95. Liu X, Uchiyama M, Kim K, et al. Sleep loss and daytime sleepiness in the general adult population of Japan. Psychiatry Res. 2000;93(1):1-11.

96. Spielman AJ, Caruso L, Glovinsky P. A behavioral perspective on insomnia treatment. Psychiatr Clin North Am. 1987;10(4):12.

97. Hauri P, Chernik D, Hawkins D, Mendels J. Sleep of depressed patients in remission. Arch Gen Psychiatry. 1974;31(3):386-391.

98. Stam HJ, Bultz BD. The treatment of severe insomnia in a cancer patient. J Behav Ther Exp Psychiatry. 1986;17(1):33-37.

99. French AP, Tupin JP. Therapeutic application of a simple relaxation method. Am J Psychother. 1974;28(2):282-287.

100. Morin CM, Kowatch RA, Wade JB. Behavioral management of sleep disturbances secondary to chronic pain. J Behav Ther Exp Psychiatry. 1989;20(4):295-302.

101. Morin CM, Kowatch RA, O'Shanick G. Sleep restriction for the inpatient treatment of insomnia. Sleep. 1990;13(2):183-186.

102. Varni J. Behavioral treatment of disease-related chronic insomnia in a hemophiliac. J Behav Ther Exp Psychiatry. 1980;11:143-145.

103. Tan TL, Kales JD, Kales A, Martin ED, Mann LD, Soldatos CR. Inpatient multidimensional management of treatment-resistant insomnia. Psychosomatics. 1987;28(5):266-272.

104. Kolko DJ. Behavioral treatment of excessive daytime sleepiness in an elderly woman with multiple medical problems. J Behav Ther Exp Psychiatry. 1984;15(4):341-345.

105. Vitiello MV, Rybarczyk B, Von Korff M, Stepanski EJ. Cognitive behavioral therapy for insomnia improves sleep and decreases pain in older adults with co-morbid insomnia and osteoarthritis. J Clin Sleep Med. 2009;5(4):355-362.

106. Currie SR, Wilson KG, Pontefract AJ, deLaplante L. Cognitive-behavioral treatment of insomnia secondary to chronic pain. J Consult Clin Psychol. 2000;68(3):407-416.

107. Edinger JD, Wohlgemuth WK, Krystal AD, Rice JR. Behavioral insomnia therapy for fibromyalgia patients: A randomized clinical trial. Arch Intern Med. 2005;165(21):2527-2535.

108. Cannici J, Malcolm R, Peek LA. Treatment of insomnia in cancer patients using muscle relaxation training. J Behav Ther Exp Psychiatry. 1983;14(3):251-256.

109. Fiorentino L. Cognitive Behavioral Therapy for Insomnia in Breast Cancer Survivors: A randomized controlled crossover study. Ann Albor, MI:Dissertation Abstracts International; 2008.

110. Savard J, Simard S, Ivers H, Morin CM. Randomized study on the efficacy of cognitive-behavioral therapy for insomnia secondary to breast cancer, part II: Immunologic effects. J Clin Oncol. 2005;23(25):6097-6106.

111. McCurry SM, Gibbons LE, Logsdon RG, Vitiello MV, Teri L. Nighttime insomnia treatment and education for Alzheimer's disease: A randomized, controlled trial. J Am Geriatr Soc. 2005;53(5): 793-802.

112. Arnedt JT, Conroy D, Rutt J, Aloia MS, Brower KJ, Armitage R. An open trial of cognitive-behavioral treatment for insomnia comorbid with alcohol dependence. Sleep Med. 2007;8(2):176-180.

113. McCrae CS, Lichstein KL. Secondary insomnia: Diagnostic challenges and intervention opportunities. Sleep Med Rev. 2001;5(1):47-61.

114. Stewart R, Besset A, Bebbington P, et al. Insomnia comorbidity and impact and hypnotic use by age group in a national survey population aged 16 to 74 years. Sleep. 2006;29(11):1391-1397.

115. Zavesicka L, Brunovsky M, Horacek J, et al. Trazodone improves the results of cognitive behaviour therapy of primary insomnia in nondepressed patients. Neuro Endocrinol Lett. 2008;29(6):895-901.

116. Mendelson WB. Combining pharmacologic and nonpharmacologic therapies for insomnia. J Clin Psychiatry. 2007;68 Suppl 5:19-23.

117. Morgan K, Dixon S, Mathers N, Thompson J, Tomeny M. Psychological treatment for insomnia in the management of long-term hypnotic drug use: A pragmatic randomised controlled trial. Br J Gen Pract. 2003;53(497):923-928. 
118. Soeffing JP, Lichstein KL, Nau SD, et al. Psychological treatment of insomnia in hypnotic-dependent older adults. Sleep Med. 2008;9(2):165-171.

119. Lichstein KL, Peterson BA, Riedel BW, Means MK, Epperson MT, Aguillard RN. Relaxation to assist sleep medication withdrawal. Behav Modif. 1999;23(3):379-402.

120. Morin CM, Bastien C, Guay B, Radouco-Thomas M, Leblanc J, Vallieres A. Randomized clinical trial of supervised tapering and cognitive behavior therapy to facilitate benzodiazepine discontinuation in older adults with chronic insomnia. Am J Psychiatry. 2004;161(2):332-342.
121. Zavesicka L, Brunovsky M, Matousek M, Sos P. Discontinuation of hypnotics during cognitive behavioural therapy for insomnia. $B M C$ Psychiatry. 2008;8:80.

122. Riemann D, Perlis ML. The treatments of chronic insomnia: A review of benzodiazepine receptor agonists and psychological and behavioral therapies. Sleep Med Rev. 2009;13(3):205-214.

\section{Publish your work in this journal}

Nature and Science of Sleep is an international, peer-reviewed, open access journal covering all aspects of sleep science and sleep medicine, including the neurophysiology and functions of sleep, the genetics of sleep, sleep and society, biological rhythms, dreaming, sleep disorders and therapy, and strategies to optimize healthy sleep. The journal welcomes
Dovepress

original research, clinical \& epidemiological studies, reviews \& evaluations, case reports and extended reports. The manuscript management system is completely online and includes a very quick and fair peerreview system, which is all easy to use. Visit http://www.dovepress.com/ testimonials.php to read real quotes from published authors.

Submit your manuscript here: http://www.dovepress.com/nature-and-science-of-sleep-journal 